\title{
Gradhiva
}

GRADHIV

Revue d'anthropologie et d'histoire des arts

$24 \mid 2016$

ARTchives

\section{À l'ombre des murs palimpsestes. Les graffiti carcéraux ou faire avec les aveux de l'histoire}

In the shadows of the palimpsest walls: Prison graffiti, or dealing with the confessions of history

\section{Anne Monjaret}

\section{OpenEdition}

\section{Journals}

Édition électronique

URL : http://journals.openedition.org/gradhiva/3288

DOI : $10.4000 /$ gradhiva.3288

ISSN : 1760-849X

\section{Éditeur}

Musée du quai Branly Jacques Chirac

Édition imprimée

Date de publication : 7 décembre 2016

Pagination : 164-189

ISBN : 978-2-35744-094-4

ISSN : 0764-8928

\section{Référence électronique}

Anne Monjaret, «À l'ombre des murs palimpsestes. Les graffiti carcéraux ou faire avec les aveux de I'histoire », Gradhiva [En ligne], 24 | 2016, mis en ligne le 07 décembre 2019, consulté le 02 mai 2019. URL : http://journals.openedition.org/gradhiva/3288 ; DOI : 10.4000/gradhiva.3288 


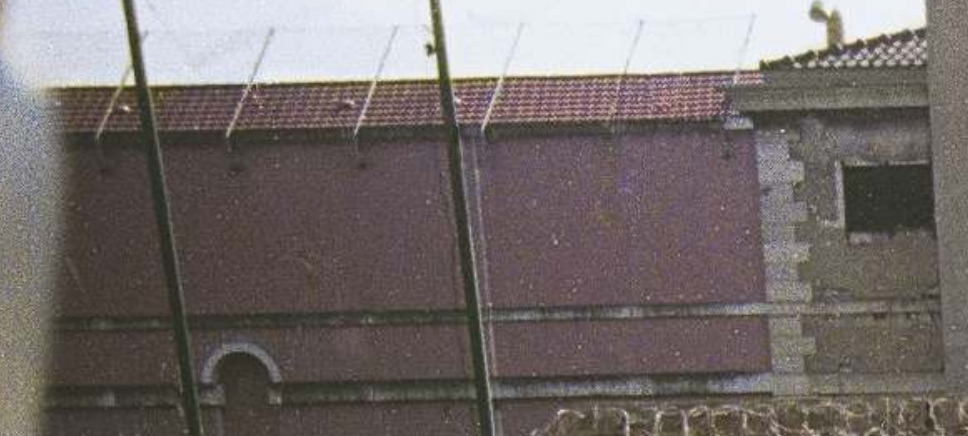

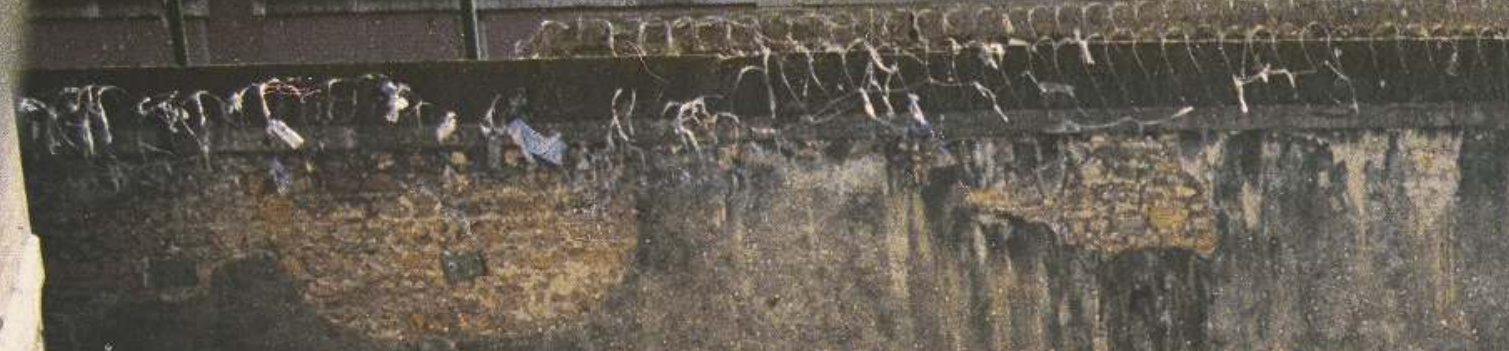

\section{Q}

起

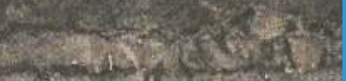

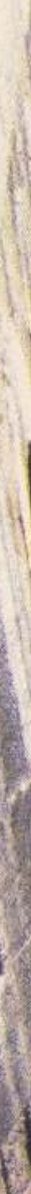

1

i

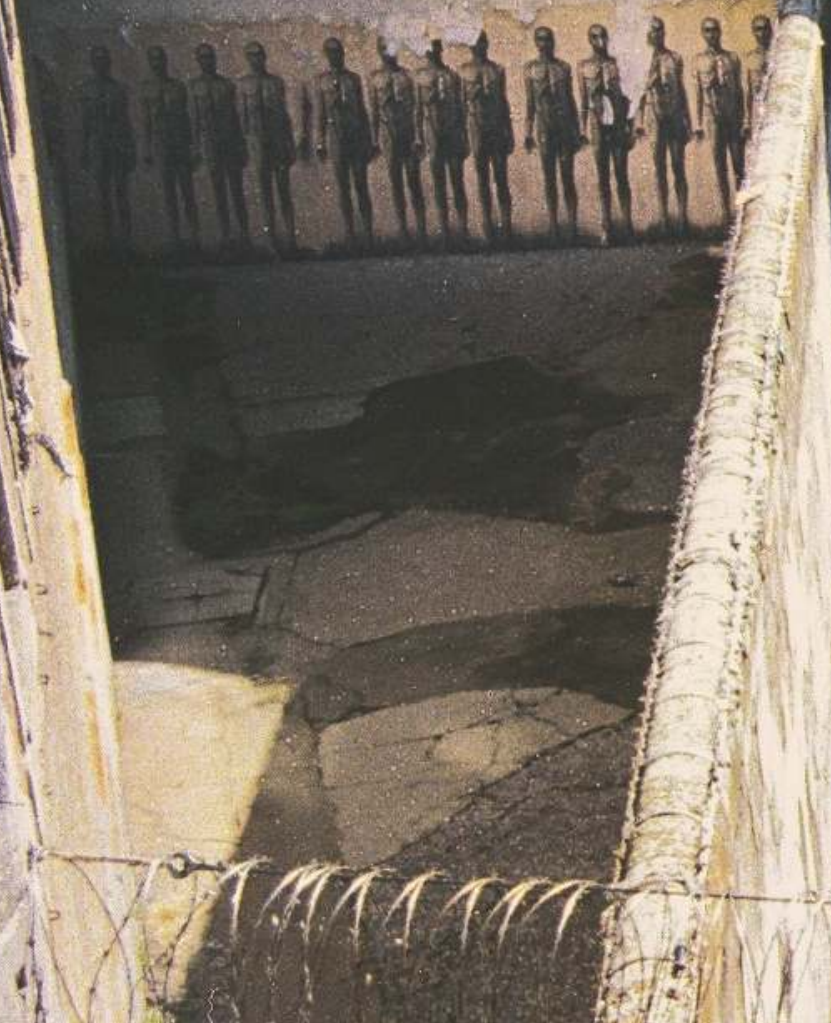

ans

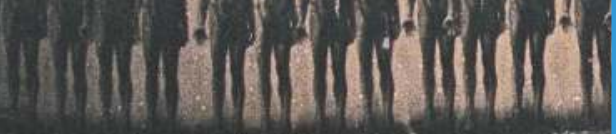

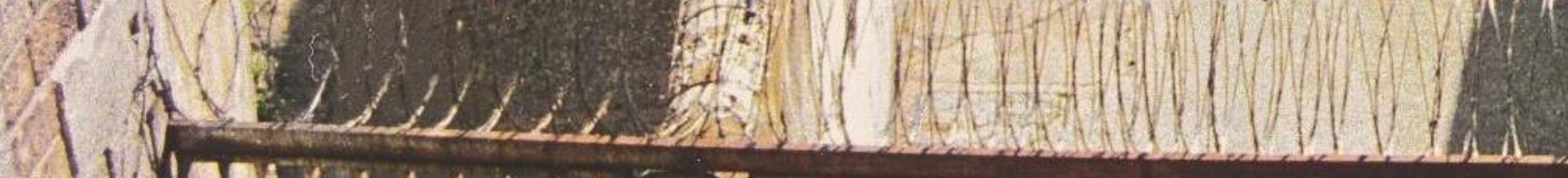

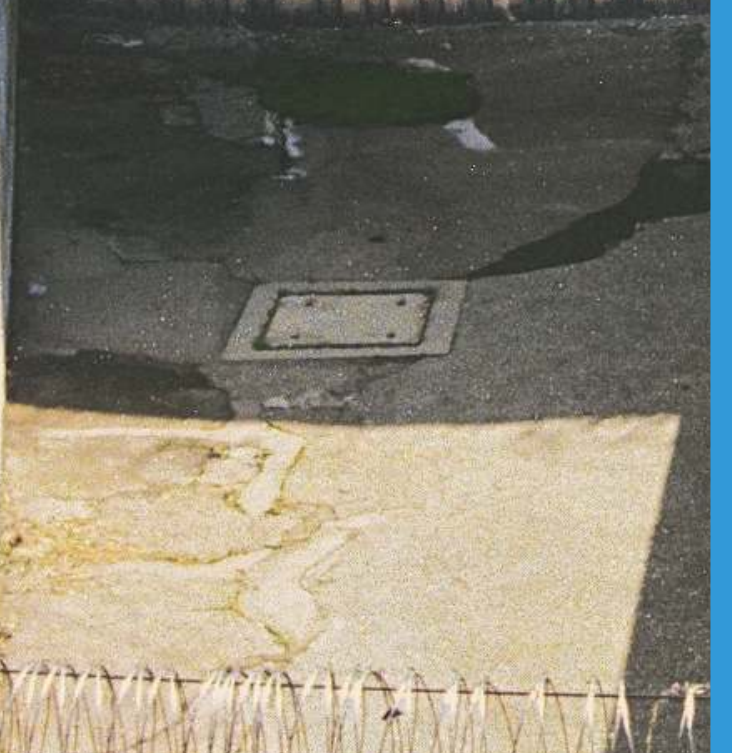

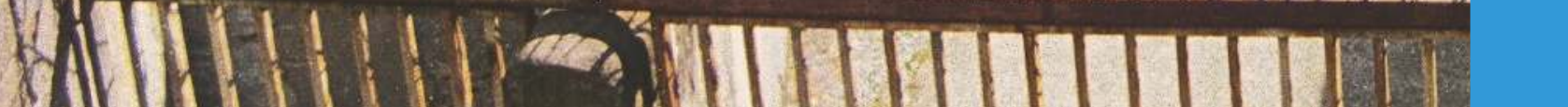
A ILIIII in

M.LL. A $111 \%$ 


\section{À l'ombre des murs palimpsestes}

Les graffiti carcéraux ou faire avec les aveux de l'histoire

par Anne Monjaret

Les graffiti de prison ont un statut qui oscille entre art et archives. Selon le regard porté sur eux, ils deviennent l'un ou l'autre, quand ils ne sont pas considérés comme les deux à la fois. Les murs carcéraux se transforment ainsi en documents à conserver ou en œuvres à montrer. Le propre de ces graffiti clandestins est de renvoyer à des faits marqués par le sceau d'une histoire sociale et politique. Anne Monjaret se propose d'interroger les manières de traiter ces « héritages encombrants ", reflets des relations institutionnelles à l'histoire. Elle analyse le rôle des ethnologues et des historiens qui composent avec ces traces murales et participent à la valorisation historique, patrimoniale et artistique de ces productions sensibles. Dans un même temps, elle s'intéresse à la place des nouveaux outils offerts par la numérisation et Internet dans ce processus. 


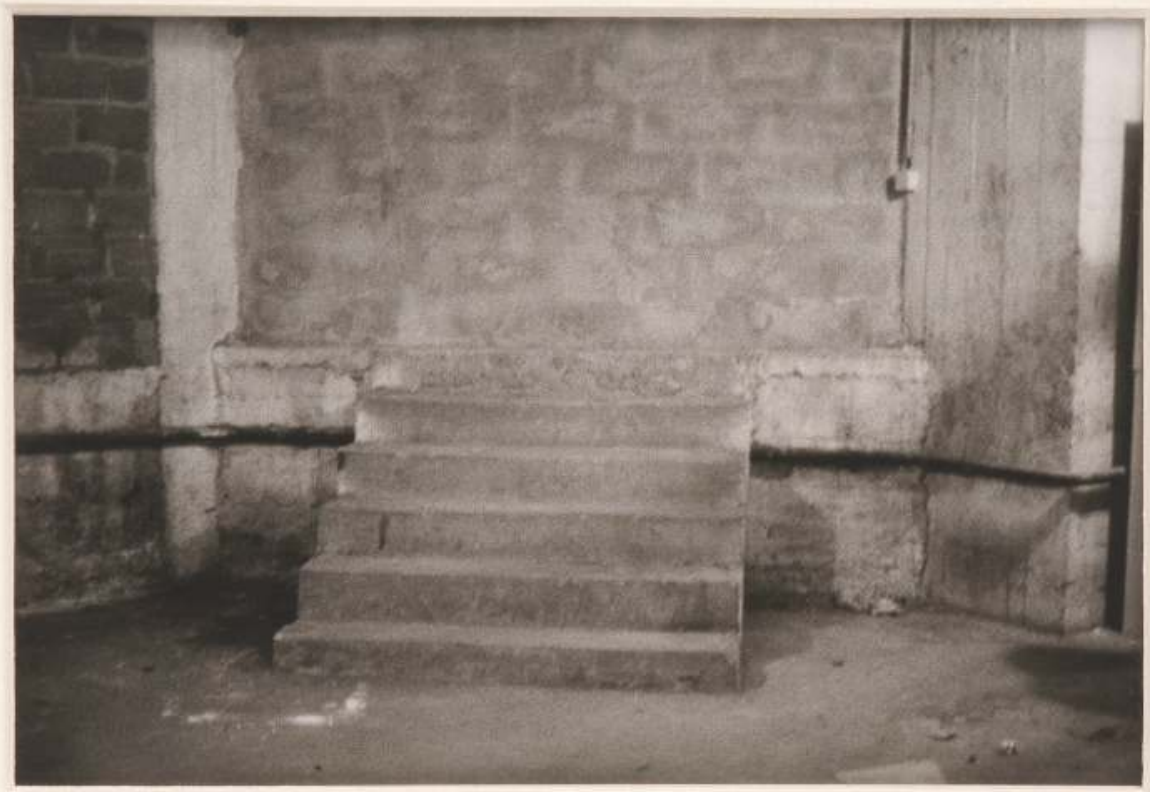

fig. 1

Bernard Plossu, Musée

national des Arts d'Afrique

et d'Océanie, escalier

dont l'issue est murée,

1985-2001, tirage sur

papier baryté, $30 \times 35 \mathrm{~cm}$.

Coll. musée du quai Branly

(c) Bernard Plossu. 


\section{À Daniel Fabre}

Les lieux de détention ne sont pas tous, dès leur naissance, des prisons (Foucault 1975): châteaux, immeubles d'habitation, hôtels, musées, etc., se sont vus transformés en espaces d'incarcération et leurs murs portent les traces déposées par les hommes qui y ont été enfermés. Celles-ci sont les indices tangibles de faits de réclusion. Ainsi, «aux côtés des graffiti de victimes politiques demeure la grande masse des expressions graphiques laissées par des détenus de droit commun [...]» (Vimont 2008: 198).

Les stigmates appartiennent à un langage commun et clandestin reconnaissable: mots, dates, chiffres, dessins tapissent souvent les murs des cellules. Avec le temps, quand elles ne sont pas effacées, ces traces peuvent se transformer en archives, en art populaire et en patrimoine. Les prisonniers deviennent alors en quelque sorte des artistes post mortem, et les graffiti carcéraux, des témoignages, ayant une valeur à la fois artistique et patrimoniale.

Cet article interroge ces changements de statut induits par une volonté de conservation et de publicisation, qui a conduit à la reconnaissance d'un art longtemps considéré comme mineur, ainsi que le rôle d'Internet dans cette valorisation patrimoniale et/ou artistique. II s'agit surtout de comprendre la façon dont se noue, à travers ces initiatives, la relation que nous entretenons avec ces héritages graphiques, témoins le plus souvent d'un passé douloureux, mais aussi de saisir le sens de ces graffiti pour leurs auteurs comme pour ceux qui en sont les héritiers, criminologues ou folkloristes et plus tard ethnologues ou historiens qui ont œuvré et œuvrent encore à les faire connaître.

L'analyse s'appuie sur plusieurs exemples de lieux carcéraux, principalement en France et en Italie, issus de travaux de seconde et de première main: des prisons ayant aujourd'hui cessé leur activité et dont certaines sont devenues des espaces culturels éphémères, parfois des musées ${ }^{1}$, et une cour à ciel ouvert du palais de la Porte Dorée à Paris, ancien musée des Colonies datant de 1931, ayant servi à un moment de son histoire d'espace carcéral. C'est en 2002, alors que je menais avec des collègues une enquête sur la mémoire des personnels du musée national des Arts d'Afrique et d'Océanie (MAAO), installé au Palais et dont les collections s'apprêtaient à le quitter (notamment Eidelman et al. 2002; 2003), que j'appris l'existence de graffiti sur les murs de cette cour.

Une attention particulière sera donc portée à la valorisation patrimoniale de ces expressions graphiques dans les prisons autant qu'à ses prolongements sur Internet (blogs privés, sites institutionnels). Le site du musée de l'Histoire de l'immigration et la plateforme scientifique Criminocorpus - comprenant une rubrique «Musée $\mathbf{2}$ - seront privilégiés. Ceux-ci montrent l'intérêt grandissant pour les graffiti carcéraux ainsi que les modalités nouvelles et plurielles permettant de composer avec ces derniers et «faire» avec notre histoire.
1. Parmi les nombreux cas relatés dans la littérature ethnologique, j'aborderai plus particulièrement, pour la France, celui de la prison de Brignoles dans le Var, site aujourd'hui désaffecté, qui a fait l'objet de plusieurs publications et dont des photographies ont été déposées sur le site criminocorpus.org. J'ai trouvé ainsi matière à réflexion sur un terrain que je n'ai pas effectue (Hameau 2004, 2008, 2013b; Truchi 2004).

2. Cette rubrique offre au public des internautes des expositions sur I'histoire carcérale et des visites en ligne des établissements pénitentiaires fermés, et consacre un volet "La mémoire des murs" aux graffiti : https:// criminocorpus.org/fr/ musee/ (consulté le 22 mars 2015). 
3. "Pareti come fogli."

\section{Les “livres de pierre" en milieu carcéral}

Mais de quelles traces s'agit-il? Des inscriptions et des dessins de toutes sortes, apposés en surface ou gravés, qui viennent noircir les murs des cellules et les faire ressembler à une page d'écriture griffonnée. Les murs peuvent devenir des «scènes du texte» (Charuty 2008), ils sont comme du papier ${ }^{3}$, nous dit Duccio Demetrio (2007: 71), sur lequel les détenus peuvent s'exprimer librement: écrire, dessiner, compter, se conter, manier à leur façon l'art du trait, parfois grossier, naïf et enfantin, donnant à voir ou à lire une part d'eux-mêmes, de leurs aspirations, de leurs revendications.

La situation d'enfermement contraint en effet à un entre-soi et souvent à un tête-à-tête avec soi. II ne s'agit pas seulement de tuer l'ennui en investissant les parois de sa cellule, mais de s'approprier un territoire intérieur, clandestinement, discrètement ou plus démonstrativement, de le faire sien en apposant une signature indélébile, et marquer son passage. «Les détenus reconstruisent leur image par des mots, des phrases et des figures qui leur permettent de se raconter. » (Hameau 2008: 160) Pour le reclus, ces signes "pariétaux» sont des repères individualisés du temps qui s'écoule comme de sa propre histoire, des «écritures du moi » révélatrices de la «tentation autobiographique» des prisonniers (ibid: 152). Philippe Hameau et Sandrine Truchi rapportent le cas exemplaire de Raymond Balestra, sourd-muet, arrêté par la Gestapo et incarcéré dix-huit jours à la prison de Brignoles en 1944 - une prison construite en 1840 et active jusqu'en 1950. Ayant appris le dessin, il s'en sert pour dérouler graphiquement des bribes de sa vie, mêlant faits tirés de son quotidien et de son imagination (Truchi 2004; Hameau 2008). Les motifs retrouvés dans les cellules n'ont pas toujours la même exceptionnalité.

Même modestes, ils agissent comme un appel de l'extérieur et un rappel de cet extérieur passé et présent. Le graffiti de prison comme tout graffiti en situation de solitude est souvent phatique. À la prison de Brignoles sont réitérés les «Allô... Allô? ». L'extérieur est ramené à l'intérieur pour mieux en sortir. Les jours mémorables sont également consignés, qui relatent une actualité ou un fait marquant au sein de la prison. Les inimitiés sont criées sur les parois et nous rappellent les difficultés d'une vie de groupe imposée, réglementée et surveillée. D'autres motifs mettent en scène des personnages au travers de portraits plus consensuels.

Ils sont surtout l'occasion de revenir sur des souvenirs que les détenus cherchent à préserver, face à l'effet possible d'effacement voire de brouillage que la réclusion tend à provoquer. "Souvenirs de " est une expression récurrente, elle se retrouve d'ailleurs sur les murs de la cour du palais de Porte Dorée où j'ai mené mon enquête. Baptisée «cour des prisonniers" par les personnels techniques, celle-ci aurait servi, d'après eux, au moment de l'Exposition coloniale de 1931, à l'incarcération de «camelots » (vendeurs à la sauvette), accusés de troubler l'ordre public. Des dires que viendront confirmer les archives: "[...] tandis que des inscriptions en chinois pourraient attester de la présence de ces "originaires d'Extrême-Orient" ", mentionnés dans le rapport général de l'exposition, qui vendaient des «bronzes ou statuettes " sans autorisation. Ces vendeurs ambulants étaient enfermés 


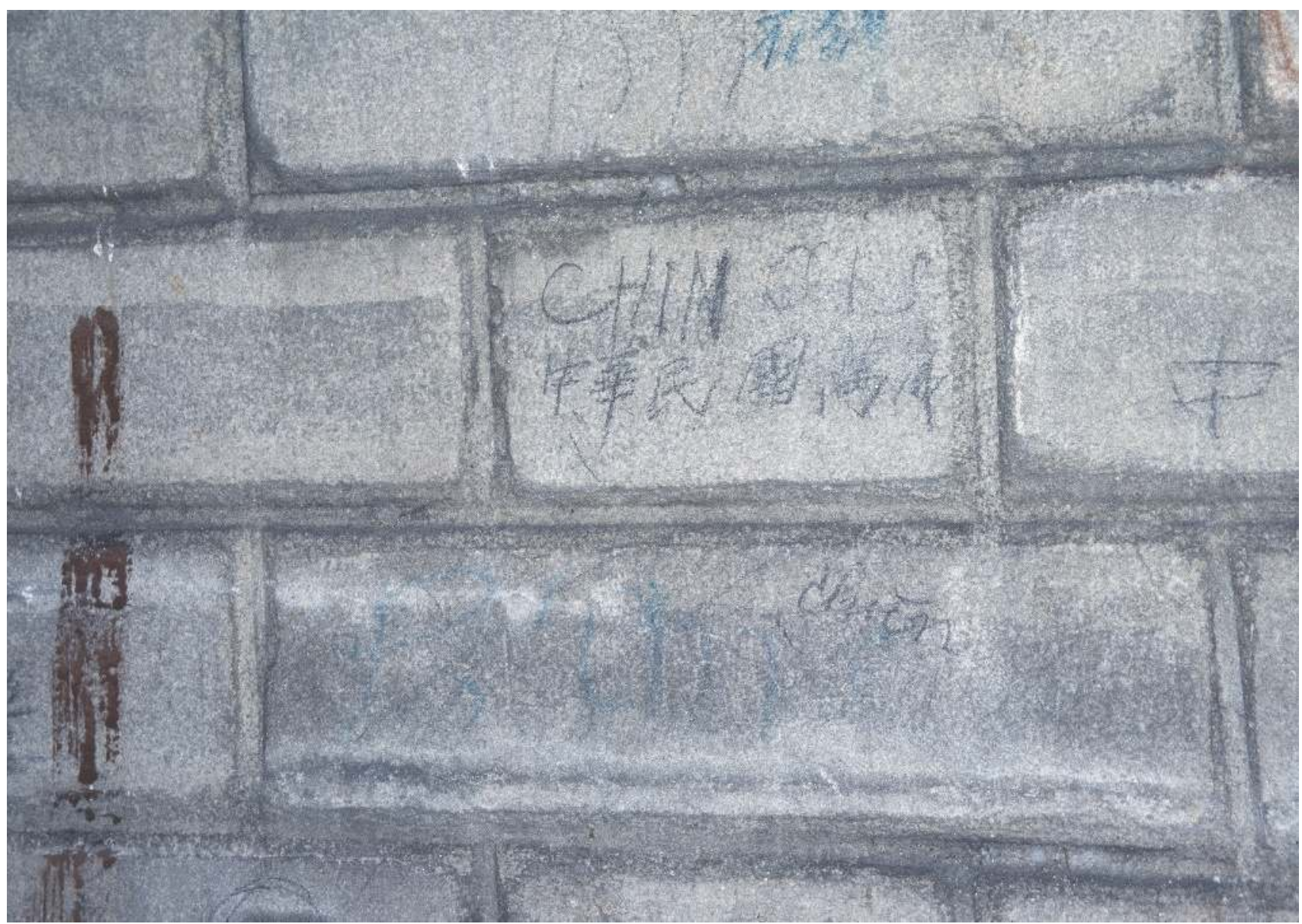

fig. 2

Graffiti sur un des murs de

la cour du musée national

des Arts d'Afrique et

d'Océanie, 2002 @) Anne

Monjaret. 
4. Extrait tiré du rapport général du gouverneur Olivier daté de 1933 - dont le chapitre I est consacré notamment à l'" utilisation de la main-d'œuvre sur les chantiers " - cité par Maureen Murphy (2007b: 55).

5. Pour un inventaire de ces graffiti, voir Monjaret 2004: 79-82.

6. Certaines personnes rencontrées évoquent une possible utilisation de la cour durant la Seconde Guerre mondiale.

7. J'entends ici par archive ordinaire et exemplaire une source historique ici une trace graphique dont le contenu renvoie à la petite histoire personnelle des individus autant qu'à l'histoire commune et partagée, voire à la Grande Histoire.

8. Traduit en français en 1894 sous le titre Les Palimpsestes des prisons", Paris, G. Masson/Lyon, A. Storck. La diffusion de ce travail se fait donc très vite au-delà des frontières italiennes.

9. Voir notamment Suzanne Stewart-Steinberg sur les approches et conceptions, à la fois convergentes et distinctes, du «peuple italien " (quid d'un atavisme) de ces deux hommes (StewartSteinberg 2007). Pierpaolo Leschiutta voit dans Lombroso un précurseur de l'anthropologie urbaine ancrée dans la modernité, alors que les folkloristes sont, selon lui, plutôt tournés vers le passé, à la recherche des signes d'une ruralité populaire originelle (Leschiutta 1996). "dans les locaux ménagés à cet effet dans les sous-sols du musée permanent 4 ". "La préfecture de police», lit-on dans les ordonnances du 22 mai et du 24 juillet 1931, «relève un total de 39500 arrestations, soit une moyenne d'environ 200 par jour ». II s'agissait d'« indigènes originaires de l'Afrique du Nord offrant des cacahuètes [...], ou encore de marchands de casques coloniaux [...], de pêches ou d'oranges" (Murphy 2007a: 36). Ces traces situées en extérieur ont passé le temps sans trop de détériorations, même si quelques-unes sont devenues plus difficiles à déchiffrer (Monjaret 2004). Ces expressions ressemblent à celles trouvées sur d'autres sites de détention: portraits de femmes à la coupe au carré, ouvrier avec casquette et clope au bec, homme au nez busqué, tête de mort, Bugatti, hiéroglyphes, dates, signatures, phrases, etc. ${ }^{5}$ renvoient principalement à la période d'occupation du lieu en 1931. D'après certains enquêtés, d'autres pourraient être le fait d'ouvriers du chantier de construction de l'édifice ${ }^{6}$.

Progressivement, le temps qui passe aura raison de ces traces personnalisées, les anonymisant et les désindividualisant, les renvoyant par là à un passé commun. Quelle que soit la durée d'emprisonnement, des motifs graphiques semblent se répéter, donnant à ces écritures marginales et populaires la teneur d'archives à la fois ordinaires et exemplaires ${ }^{7}$. Comment l'intérêt pour ces expressions graphiques va-t-il émerger et pour quelles raisons? Comment évolue-t-il? Quels sont les rapports que nous entretenons avec cet héritage emblématique des lieux de l'enfermement?

\section{Prémices d'un intérêt pour les écritures clandestines}

Les premières initiatives de collecte et d'inventaire de graffiti, qui remontent au $x \mid x^{e}$ siècle, n'ont pas une vocation à proprement parler patrimoniale. On doit l'exemple le plus connu à l'Italien Cesare Lombroso, fondateur de l'anthropologie criminelle (Leschiutta 1996; Portigliatti Barbos 2004; Fabre 2007). Ce dernier entreprend notamment l'inventaire des graffiti de prison trouvés sur les murs, les meubles, les poteries. Cette collecte lui permet de mettre à nu l'âme ou plus exactement les états d'âme des détenus en dressant des types de «physionomie» et de pathologie. L'inventaire qu'il publie en 1888 dans un ouvrage intitulé Palimsesti del carcere ${ }^{8}$ est destiné à nourrir ses travaux en criminologie. Comme le précise Daniel Fabre: «[...] Lombroso ne lit pas le livre de pierre avec l'œil de l'historien en quête de nouvelles archives» (Fabre 2007: 52, traduction de Daniel Fabre), mais avec l'œil du criminologue qui cherche à y déceler «la tare de la délinquance». Même si ses préoccupations étaient d'ordre psychosociologique et non esthétique, il reste un «collectionneur frénétique» fasciné et ému par ce qu'il avait sous les yeux.

II n'est pas le seul Italien à s'être intéressé au tournant du xxe siècle à ces traces graphiques que l'on trouve sur les murs des lieux de détention. Giuseppe Pitrè, folkloriste, fondateur de l'ethnographie sicilienne, découvre en 1906 avec stupéfaction sur les murs du palais Chiaramonte à Palerme les plaintes et supplications laissées par des victimes du tribunal de l'Inquisition. Comme le rappelle Giordana Charuty, même s'il est tout autant bouleversé que Lombroso, la démarche ethnographique de Pitrè comme ses interprétations diffèrent toutefois de celles du criminologue, au regard plus clinique ${ }^{9}$. Ce dernier va s'attacher pendant six mois - insiste Giordana 
Charuty - à gratter les murs des geôles marquées par la profusion de ces «enchevêtrements de signes graphiques et plastiques» (Charuty 2006: 26). «Sous les couches de chaux qu'il décolle avec précaution, des strates successives de prières, de suppliques, de maximes et de poèmes entrelacés aux figures de saints, d'orants, de paysages transforment toutes les surfaces accessibles en d'immenses "palimpsestes" dont Pitrè entreprend aussitôt le relevé, d'une remarquable précision [...]. Pitrè s'emploie à faire surgir des sujets écrivants [...]. Poursuivie dans d'autres archives, l'enquête arrachera à l'anonymat quelques-unes de ces "âmes qui crient" [...]. . (Ibid. : 26) Cette recherche, tombée dans l'oubli, ne sera publiée qu'en 1940 de façon posthume; puis dans les années 1960, alerté par le journaliste Giuseppe Quatriglio, l'écrivain Leonardo Sciascia, soucieux des injustices judiciaires, découvre à son tour le site sicilien. II réalise dans l'urgence des photographies qui alimentent un premier livre paru en 1977. Mais ce n'est qu'en 1999 que le dossier trouve une nouvelle visibilité grâce à la réédition augmentée de l'ouvrage (Pitrè et Sciascia 1999). Sa fille, Laura Sciascia, continue aujourd'hui le travail de son père et le palais Chiaramonte de Palerme a ouvert ses portes au public, donnant à voir les graffiti qui ont pu y être conservés. Pitrè aura contribué, à sa manière, à la patrimonialisation de ces traces du passé.

Ces deux exemples italiens permettent d'éclairer une configuration particulière de savoirs et, partant, d'acteurs: on se trouve en présence, d'une part, d'une science médicale qui produit «son art» en inventant une documentation systématique de type archivistique et, d'autre part, d'une ethnographie qui localise un art peinant cependant à être publicisé. Ces différences disciplinaires n'effacent pas, bien au contraire, la perception commune des graffiti de prison, à savoir qu'ils sont et restent les expressions du drame et de la douleur des prisonniers.

En France, comme Jean-Claude Vimont le rappelle sur le site de Criminocorpus ${ }^{10}$, parmi les premières initiatives il faut noter, après la Seconde Guerre mondiale, la collecte - à l'initiative du ministère des Prisonniers, Déportés et Réfugiés - des graffiti des résistants incarcérés à Fresnes, devenue prison allemande sous l'Occupation, afin d'identifier des disparus (Calet 1993 [1945]). Les traces graphiques correspondent ici davantage à une source d'information qu'à un patrimoine à sauvegarder. Stigmates d'une histoire sensible, elles deviennent une preuve des exactions de la guerre. On peut se demander si ce n'est pas la difficile confrontation avec ces aveux de l'histoire résultant d'épisodes particulièrement tragiques qui nous ont rendus aveugles, sinon peu réceptifs à leur existence. Autrement dit, le graffiti de prison plus qu'un autre gêne, parce qu'il nous confronte à la situation difficilement compréhensible de l'incarcération.

On comprend mieux alors pourquoi le chemin de cette valorisation patrimoniale a pu être long et tortueux. Selon Philippe Hameau, «les «graffiti de prison ne constituent pas une thématique très fréquente dans la recherche anthropologique alors que l'univers carcéral y est couramment analysé» (Hameau 2013b: 85). Daniel Fabre rappelle quant à lui «[...] que ces cris silencieux mettront exactement un siècle pour être reconnus comme un bien collectif précieux par l'administration culturelle et donneront lieu à un
10. https://criminocorpus. org/fr/musee/la-memoiredes-murs/ (consulté le 9 mai 2016). 


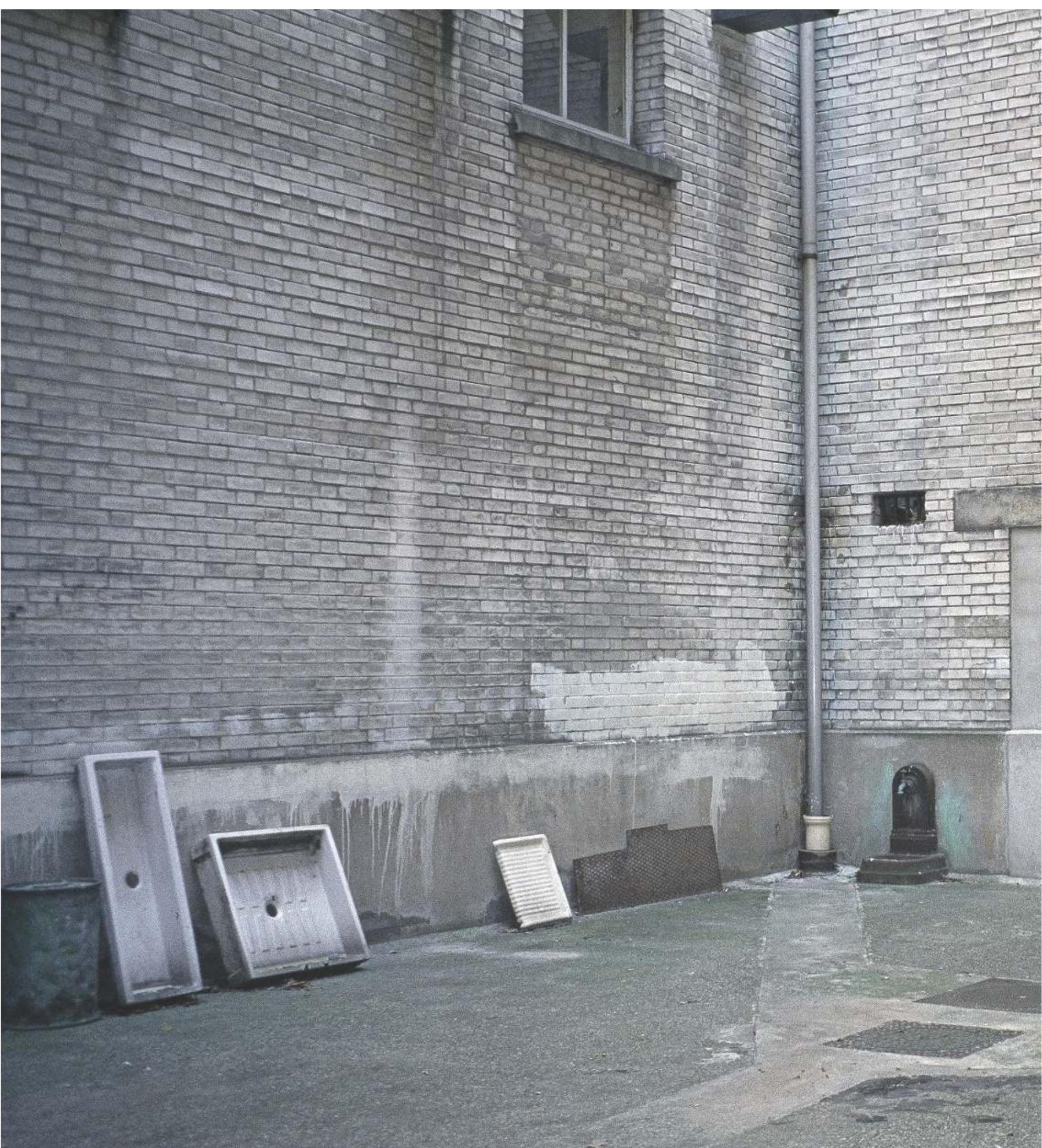




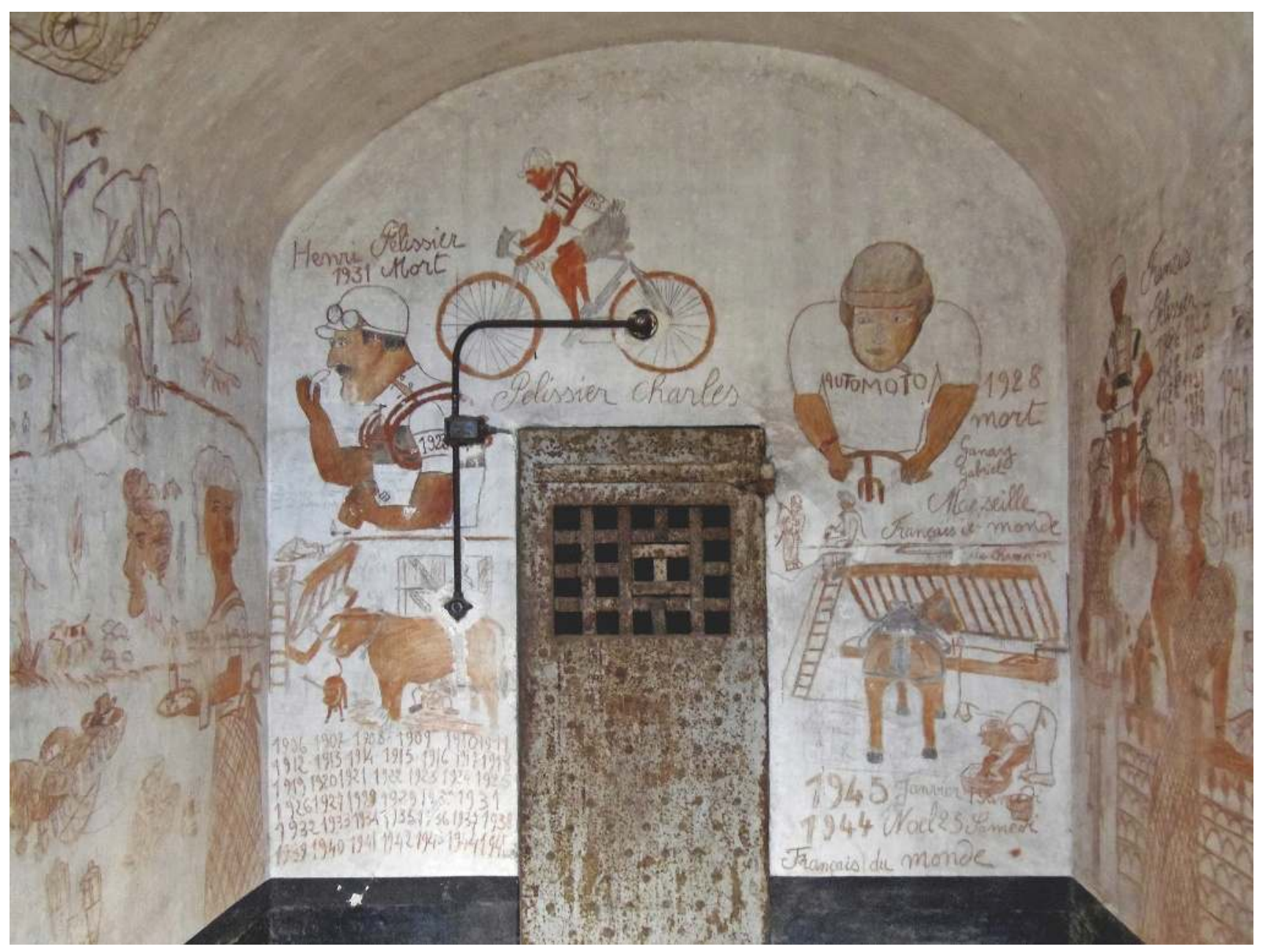

fig. 4

Les Cyclistes, graffit

à la prison de Brignolles,

v. 2004-2006 () Philippe

Hameau. 
combat patrimonial des plus animés auquel participeront des intellectuels de haut vol [...] » (Fabre 2007 : 55, traduction de Daniel Fabre). En effet, il faut attendre les années 2000 pour qu'ils fassent l'objet d'un engouement certain et toujours plus prononcé. II suffit de lister quelques travaux en Europe (en France, Italie, Espagne) pour s'en convaincre (Benavente et al. 2004; Candau et Hameau [dir.] 2004; Clemente [dir.] 2007; Fabre 2007; Hameau 2004, 2008, 2009a et b, 2013a et b). C'est ainsi que les historiens comme les ethnologues sont devenus les passeurs de mémoire de ces productions carcérales. «La constatation s'impose de nouveau: c'est au moment où une culture n'a plus les moyens de se défendre que l'ethnologue ou l'archéologue apparaissent. » (Certeau 1993 [1974]: 51) Cet intérêt tient à la prise de conscience de la fragilité de ces traces. Pour Jean-Claude Vimont, il devient urgent de se préoccuper de ces «graffiti en péril », de venir à leur rescousse avant qu'il ne soit trop tard. «Parmi les sources de l'écriture de l'histoire, il en est de plus fragiles que d'autres, menacées par leur matérialité même, négligées au profit de recueils documentaires plus riches, reléguées dans la sphère des annexes iconographiques. » (Vimont 2008: 195) L'auteur s'inquiète de l'état précaire de ces archives peu valorisées et alerte l'opinion publique sur la menace de leur perte définitive. Quel futur leur est-il réservé? Sont-elles vouées à l'oubli ou dignes de notre souvenir? Allons-nous les effacer ou les valoriser? Quelles stratégies mémorielles et patrimoniales peut-on observer? Qui en sont les initiateurs?

\section{La tentation de conservation ou} une patrimonialisation minimale

La fermeture d'un site carcéral conduit à se poser la question du devenir des graffiti qu'il abrite: abandon, destruction, restauration, valorisation, etc. Leur destinée se fait plurielle, mais pour certains historiens, ethnologues ou encore amateurs de vestiges historiques, seule leur sauvegarde importe. Celle-ci apparaît d'autant plus nécessaire que les pages griffonnées de ce «manuscrit» pariétal périssable apparaissent comme un «trésor» archivistique précieux. "Sources fragiles, ils [les graffiti] exigent collectes et reproductions, moulages et photographies avant que ne disparaissent avec la fermeture de nombreux établissements les traces d'une présence et les témoignages d'un vécu. » (Ibid. 2008: 202)

Les ethnologues qui ont compris les enjeux mémoriaux de cette préservation font face à l'absence d'intérêt des institutions locales, en tentant à leur manière d'œuvrer au devoir de mémoire, jusque-là déficient. Ils cherchent à faire connaître ces empreintes graphiques, à les sortir de la clandestinité et parfois à donner des noms à leurs auteurs, ce que les collectivités devenues propriétaires de ces établissements carcéraux désaffectés ne veulent ou ne peuvent assumer.

Ainsi, Philippe Hameau et ses collègues ont entrepris une étude de fond de la prison de Brignoles ${ }^{\mathbf{1 1}}$, aujourd'hui désaffectée. Leur campagne a bénéficié de l'aide de l'Association de sauvegarde, d'étude et de recherche du Centre-Var (Aser). "La quantité des graffiti observés dans l'ancienne prison de Brignoles permet d'en dresser un palmarès thématique » (Truchi 2004: 29) et souligne surtout l'exceptionnalité du corpus. L'équipe a visité les trente-six
11. D'autres initiatives de ce type existent ailleurs en France, mais elles restent encore timides. 
12. Une première enquête a été menée entre 2001 et 2002 avec Jacqueline Eidelman et Mélanie Roustan, à la veille de la fermeture du MAAO. Par la suite, j'ai poursuivi seule un moment les investigations sur la cour du Palais, alors que la $\mathrm{CNHI}$ se préparait à ouvrir. En 2011, Mélanie Roustan et moi avons repris les recherches en nous concentrant sur les transformations professionnelles au sein du musée, puis plus récemment sur la place des artistes contemporains au musée national de l'Histoire de l'immigration. cellules sur les trois niveaux du bâtiment, effectuant des relevés des graffiti, les photographiant et les décrivant. Puis les données recueillies sur le site furent croisées avec des documents d'archives. Ce travail d'inventaire, de quantification et d'analyse fut suivi de plusieurs publications (notamment Hameau 2004 et Truchi 2004), comme autant d'antidotes à l'abandon et à l'oubli. Le numéro "Cicatrices murales » de la revue Le Monde alpin et rhodanien en est un exemple: «À l'invention succède souvent la patrimonialisation, à laquelle participe d'ailleurs ce numéro [...]. Toutes [les contributions] montrent l'intérêt que peuvent avoir les chercheurs à inventorier les graffiti de prisons et à les constituer en corpus à des fins de sauvegarde - ces traces sont éminemment périssables et souvent menacées - et de comparaison. " (Candau et Hameau [dir.] 2004: 10-11) À défaut des graffiti eux-mêmes, il nous reste leur trace, autrement dit, la trace de ces traces.

II arrive que l'ethnologue, pris par sa recherche, rate en quelque sorte sa mission patrimoniale. Telle a été mon expérience au début des années 2000 lors des enquêtes ethnographiques menées au moment de la fermeture du MAAO puis de l'ouverture de la Cité nationale de l'histoire de l'immigration (CNHI) ${ }^{\mathbf{1 2}}$. En 2002, un des ouvriers rencontrés sur le site décide de me montrer la fameuse cour des prisonniers, située dans les sous-sols du bâtiment, qu'il connaît bien puisqu'elle jouxte son atelier. Il a eu le temps d'examiner dans le détail les graffiti inscrits à hauteur d'homme sur ses murs, si petits parfois qu'on ne les voit qu'au prix d'une extrême attention. Lui et ses collègues se sont en quelque sorte approprié la cour, mais ce n'est pas pour autant qu'ils l'investissent fortement; elle appartient à leur décor. Ils s'y rendent, mais ils ne m'ont jamais dit ce qu'ils y faisaient réellement. Des anciens - gardiens bénéficiant d'emplois réservés aux militaires à la retraite, vétérans de la marine ou de l'armée de terre - leur ont rapporté des fragments d'histoires du lieu dont ils sont aujourd'hui les dépositaires et qu'ils me font partager. Étrangère à leur univers et attentive à leurs témoignages, sans doute leur suis-je apparue comme un interlocuteur idéal, pouvant jouer le rôle de passeur. La fermeture annoncée du MAAO aura précipité, d'une part, une prise de conscience de la portée historique des graffiti laissés dans cette cour par les «camelots » incarcérés en 1931 et, d'autre part, la nécessité de leur divulgation.

Ce n'est qu'une année plus tard que j'entreprends d'inventorier les graffiti de la cour, de prendre des photographies et de recueillir des témoignages, travail qui a abouti à la rédaction d'un article titré «La cour des prisonniers» (Monjaret 2004). Au moment de sa publication, fin 2004, je ne mesure pas encore l'impact qu'il aura ni les rebondissements successifs qui suivront, même si j'ai conscience que sa parution participe au processus de publicisation de ces graffiti, en les portant à la connaissance de tous et en les réinscrivant dans le cours de l'histoire. Mais c'est l'histoire sombre et encore taboue des années coloniales qui remonte à la surface. En 2006, j'envoie donc mon article à la direction de la $\mathrm{CNHI}$. La réponse ne se fait pas attendre devant l'étonnement suscité par son contenu. À défaut de conservation in situ, une campagne photographique couverte par Awatef Chengal, l'un des médiateurs du musée, est menée in extremis. La photographie apparaît ici comme le dernier rempart contre la perte, une réparation de la dette par une patrimonialisation minimale. 
Mon article circule très vite au sein de l'établissement. Maureen Murphy, l'historienne de l'art qui prépare l'exposition inaugurale, en recoupe les données avec celles qu'elle a trouvées incidemment dans des archives liées à l'Exposition coloniale de 1931, confirmant l'enfermement de «camelots». Mais, à ce moment-là, le chantier de modernisation du Palais est entamé, et la cour condamnée. Je m'y rends la veille du démarrage des travaux. Je n'y retournerai ensuite qu'en 2014, et ne peux alors que constater les dégâts occasionnés par la mise aux normes du bâtiment - seuls quelques rares graffiti ont survécu. Je regrette alors de ne pas avoir moi-même réagi plus tôt pour entamer la sauvegarde du site, manquant pour ainsi dire un rendezvous avec l'histoire.

En 2007, dans le catalogue rédigé à l'occasion de l'ouverture de la CNHI (Murphy 2007a) ainsi que dans un article publié dans la revue Hommes \& Migrations (Murphy 2007b), un court développement est consacré à la «cour des prisonniers ». Ces publications du musée dédiées à l'histoire du lieu et à son devenir officialisent la reconnaissance institutionnelle de l'existence des graffiti et la prise en considération publique de son héritage colonial. Avec cette même intention, en 2013, est inauguré un «Parcours historique du palais » - qui a suscité des débats vifs et contrastés en interne -, dont l'une des tablettes numériques proposée aux visiteurs évoque la cour. II aura fallu du temps à l'institution pour montrer ces graffiti alors même qu'ils se trouvaient au cœur d'un musée, et pour se saisir ainsi et plus largement de I'histoire coloniale du Palais.

\section{L'officiel et l'officieux}

Ce monument hérité de l'exposition coloniale de 1931 peine à se libérer de son histoire. Coexistent dans ses murs un art savant - l'Art déco connu et reconnu, sinon patrimonialisé, et un art populaire - les graffiti - non accessible au plus grand nombre car longtemps tenu à l'écart, voire secret. En effet, ce monument-message (Debray 1999) à la gloire du colonisateur s'impose principalement comme l'une des références majeures à l'Art déco: son architecture, ses grilles forgées, ses basreliefs extérieurs (figurant les apports des colonies à la France), ses fresques (figurant les apports de la France aux colonies), son carrelage, ses parquets et son mobilier intérieur participent d'un ensemble artistique unique. Ces caractéristiques ont autorisé une histoire du lieu valorisée par son inscription dans l'Art, faisant l'impasse, jusqu'à il y a encore peu, sur son histoire géopolitique. La scène - l'officiel et le glorieux - se distingue ainsi des coulisses - l'officieux et le honteux auxquels renvoient les graffiti de la «cour des prisonniers».

La muséification et par conséquent la patrimonialisation de ces expressions graphiques auront donc été minimales, à l'image de leur discrétion. Si l'on en croit Primo Levi (1987 [1958]) et d'autres auteurs après lui comme Octave Debary (2000), cette patrimonialisation ne peut se substituer à la cour elle-même. Tout au plus peut-elle permettre d'en faire le deuil. 


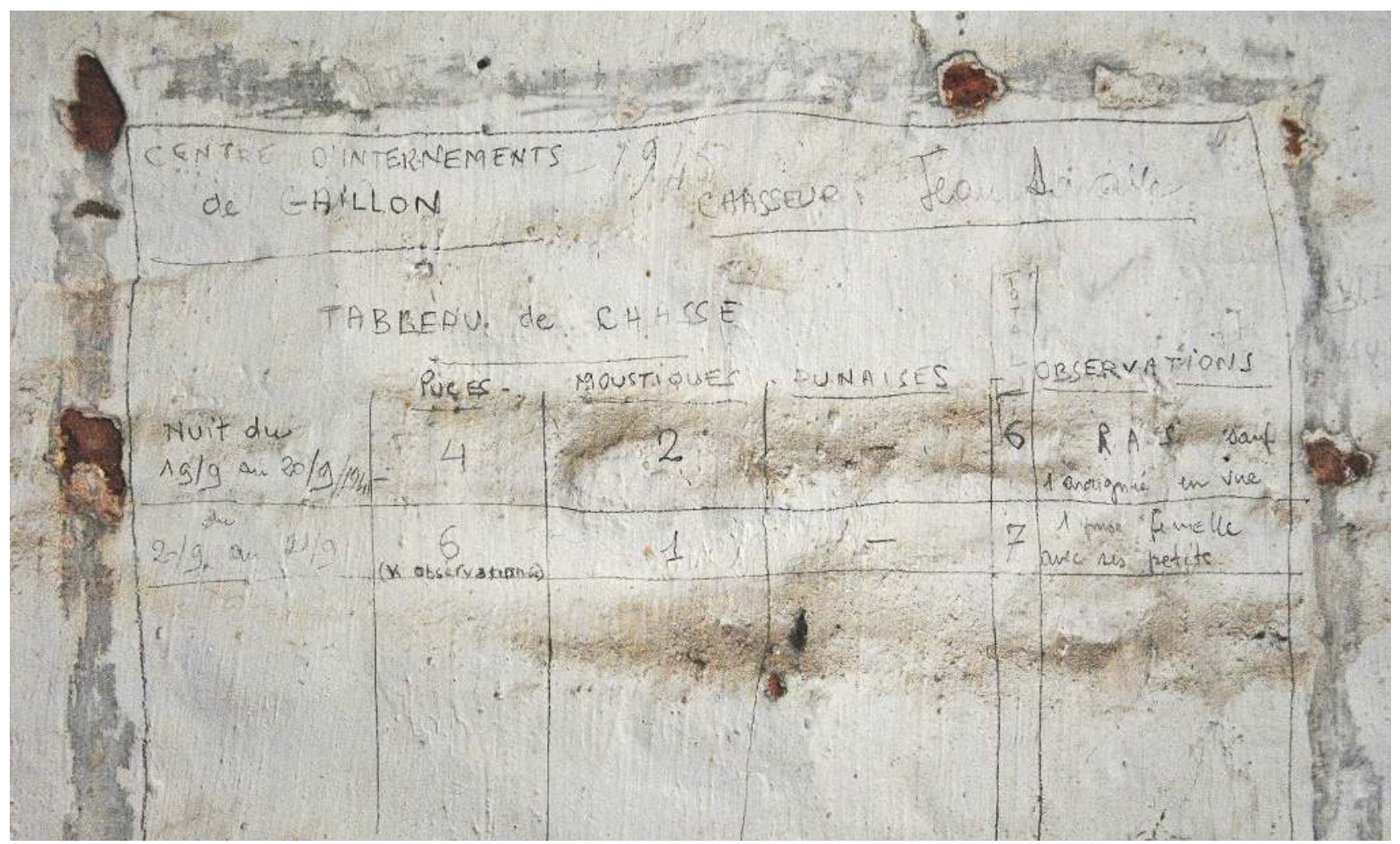

\section{fig. 5}

Compte de puces, punaises et moustiques centre de détention du château de Gaillon ( ) Drac de Normandie - Udap de l'Eure - Dominique Pitte France Poulain, 2015.
13. Sans doute l'esthétique des friches est-elle pour quelque chose dans ce nouvel engouement pour les musées éphémères qui s'implantent un temps dans un lieu en attente d'avenir ou avant une mort annoncée, l'événement sonnant alors comme un enterrement (Monjaret [dir.] 2005).
Sa non-conservation in situ aura certainement raison de la mémoire et du contexte d'exécution des graffiti qu'elle abrite. Chaque génération se retrouve de la sorte devant sa responsabilité de devoir mémoriel.

II reste que, aussi minimes soient-elles, ces dynamiques muséales ou patrimoniales auxquelles participent notamment les ethnologues contribuent au mouvement de valorisation des graffiti carcéraux et à leur changement de statut.

\section{Exposer les graffiti ou le retour sur un passé douloureux}

Les graffiti ne sont pas seulement des documents archivistiques à conserver, ils sont progressivement apparus comme des créations artistiques à montrer, un art populaire que l'on fait dialoguer avec l'art contemporain.

Mais la reconnaissance de cet art dit populaire ne s'est pas faite sans encombre: «Ces tracés sur les murs et les objets, ces compositions qui ne transmettent pas seulement un message mais s'efforcent de le rendre singulier, attractif, troublent ceux qui les découvrent par leur puissance expressive. Ruinant définitivement l'autorité des académismes, on va jusqu'à reconnaître de l'art dans ces productions marginales, issues de populations dominées jusqu'alors définies par leur déficit de culture. » (Fabre 2007: 54, traduction de Daniel Fabre)

Cette reconnaissance sort les graffiti de leur cadre intimiste. Dans d'anciennes prisons transformées momentanément en musées ${ }^{13}$ ou dans les 
prisons réhabilitées en musée, le dialogue entre les réalisations contemporaines - installations, tableaux, dessins - et celles du passé - graffiti carcéraux - devient possible.

Les murs s'exposent et servent à exposer, créant la valeur archivistique, patrimoniale et artistique de ce qui est présenté. Qu'en est-il donc du rôle des expositions d'art contemporain qui valorisent les graffiti de prison? Permettent-elles pour autant de considérer ces traces graphiques comme un art? Parviennent-elles à engager un travail de mémoire autour des histoires douloureuses qui les caractérisent?

En les donnant à voir à un large public, ces expositions ont conféré au graffiti le statut de patrimoine et d'art. Quant aux prisonniers, maîtres de ces productions, ils sont devenus à leur insu des artistes, le plus souvent anonymes, à moins que l'une de leur signature n'ait été reconnue. Leurs productions côtoient désormais celles des artistes contemporains qui, de leur côté, se saisissant de l'histoire de ces lieux carcéraux, peuvent apparaître comme des médiateurs de ce passé douloureux.

Le cas de la prison Saint-Paul à Lyon, fermée depuis 2009, est en cela intéressant. Aujourd'hui, seule la partie conservée des bâtiments dans l'enceinte du nouveau campus de l'Université catholique de Lyon témoigne de l'activité passée du site. Cette prison n'est pas n'importe quelle prison; son histoire reste fortement liée à l'Occupation. Des résistants comme Robert Namiand, Jean Moulin, Raymond Aubrac et d'autres y ont en effet été emprisonnés, torturés, voire exécutés, à l'instar d'Émile Bertrand ou Simon Fryd. En 2012, à l'occasion des Journées du patrimoine, elle fut exceptionnellement rouverte, accueillant notamment les œuvres d'Ernest Pignon-Ernest, dont certaines furent exposées à la galerie Lelong à Paris en 2014 (PignonErnest 2014). Pour Gérard Mordillat, «beaucoup sont morts à Saint-Paul, fusillés, guillotinés, suicidés; beaucoup ont laissé leurs traces sur ses murs sinistres, lépreux, puants et portent en eux l'empreinte de la prison sans jamais pouvoir s'en défaire, même une fois élargis. Ernest Pignon-Ernest les convoque dans de grands dessins installés dans les coursives, dans les couloirs, dans les cours de promenade cernées de barbelés. [...] Les corps prisonniers reprennent possession d'un espace - l'espace mémoriel, l'espace carcéral - et réintègrent une identité que le temps s'emploie à enfouir dans la fosse commune de l'oubli. » (Pignon-Ernest 2014: 14-15) Le lieu est habité aussi par ses fantômes (Fabre et luso [dir.] 2010), et si les œuvres de papier d'Ernest Pignon-Ernest semblent a priori lutter contre l'amnésie en redonnant un visage aux disparus et une humanité aux lieux, leur force esthétique ne risque-t-elle pas de reléguer au second plan les marques plus discrètes que les détenus ont laissées?

L'exposition «La disparition des lucioles» qui eut lieu à Avignon en 2014 dans la prison Sainte-Anne, construite à la fin du xvIII ${ }^{\mathrm{e}}$ siècle et désaffectée depuis 2003 (Mézil [dir.] 2014; Berthet 2015), a d'emblée été conçue en croisant art, histoire (par les archives) et mémoire (par la parole), évitant le risque de la confrontation de deux arts, l'un savant contemporain et l'autre populaire passé. Des pièces de la collection Yvon Lambert et d'autres œuvres d'artistes - dont Genet, Brassaï ou Warhol - installées dans les cours, les 
14. Aujourd'hui, l'art s'invite également dans les prisons encore en activité. Les musées proposent de partager avec les détenus des expériences culturelles (Saurier 2016). En Italie, l'association Wunder K. à Milan a conduit des personnes incarcérées à réinterpréter les traces du passé dans des ateliers d'art-thérapie (d'après une information de Giordana Charuty).

15. http://blog-chantier. histoire-immigration.fr/ ?2007/03/07/16-la-courdes-prisonniers (consulté le 15 janvier 2016). Notons qu'en septembre 2016, ce lien n'est plus actif. Seul l'accès aux pages de garde des rubriques du blog est encore possible, mais les photographies ont disparu. Cette inaccessibilité, qu'elle soit momentanée ou permanente, participe, par voie de conséquence, à l'inexorable disparition de la " cour des prisonniers". Elle montre aussi combien les usages patrimoniaux sur Internet peuvent être éphémères, et que ces sources numériques offertes à tous ne se substituent pas à la matérialité d'un fonds archivistique.

16. https://www.flickr.com/ photos/cite_immigration/ 4322074363/in/ photostream/ (consulté le 15 janvier 2016).

\section{ci-contre}

fig. 6

Bernard Plossu, Musée national des Arts d'Afrique et d'Océanie, statue d'un homme couché devant les bas-reliefs de la façade, 1985-2001, tirage sur papier baryté, $30 \times 35 \mathrm{~cm}$. Coll. musée du quai Branly (C) Bernard Plossu. couloirs et les cellules sont entrées en résonance avec les graffiti, devenus à leur tour des œuvres d'art, sans que leur portée politique et sociale ne soit négligée. Si ces initiatives culturelles carcérales valorisant l'art en prison participent d'une certaine «beauté du mort " (Certeau 1993 [1974]), elles invitent surtout les visiteurs à poser un regard sur notre histoire ${ }^{14}$.

\section{Le numérique à la rescousse des graffiti ou le danger de la perte de sens}

Il arrive cependant que les portes de ces univers carcéraux abandonnés ne s'entrouvrent plus guère ou qu'il ne soit plus possible d'y accéder. Les expositions virtuelles proposées hors les murs, sur Internet, deviennent alors l'une des solutions pour en prendre connaissance. Quels sont ces sites numériques et comment participent-ils à la valorisation des graffiti?

II semble que les initiatives - du moins celles que j'ai repérées - émanent d'institutions carcérales, muséales, juridiques et de recherche, et ce, même si elles sont nées de démarches individuelles: on retrouve une rubrique consacrée aux graffiti sur la plateforme Criminocorpus ou sur le blog "C'est le chantier» du musée national de l'Histoire de l'immigration (ex-CNHI); de même que sur le blog "Bruno des Baumettes: journal d'un détenu au quartier des "isolés", prison des Baumettes à Marseille", à la page "Des mots et des murs». Si les graffiti ne sont pas le sujet principal de ces sites, ils y occupent néanmoins une place non négligeable.

Revenons au cas de la "cour des prisonniers" du palais de la Porte Dorée, car il est un bon exemple pour interroger le rôle d'Internet dans la démarche de patrimonialisation.

Après une campagne photographique, dès le 7 mars 2007, sept clichés ont été déposés sur le blog "C'est le chantier» et adossés à la catégorie "Coins insolites ${ }^{\mathbf{1 5}}$ ": quatre d'entre elles proposent des vues globales du lieu, suivies de la légende "Vue d'ensemble de la "cour des prisonniers". Photo: Awatef Chengal $\odot \mathrm{CNHI}$ ", et trois autres montrent des vues de près des graffiti avec pour légende "Détail d'un mur de la "cour des prisonniers". Photo: Awatef Chengal ( ) CNHI». Quelques commentaires sommaires les accompagnent: "On trouve dans l'enceinte du Palais deux petites courettes intérieures. L'une d'elles a été surnommée la "cour des prisonniers" "; puis: "Rien de très particulier que cette cour au premier abord, mais en s'approchant des murs on remarque qu'ils sont couverts de graffiti: des noms, des messages, des dessins et..., ce qui semble être des idéogrammes chinois!»; et enfin: "On ne sait pas grand-chose de cette cour hormis que pendant l'Exposition coloniale de 1931 (à l'occasion de laquelle le Palais a été construit) elle aurait servi à la police de lieu de détention pour des vendeurs ambulants qui vendaient diverses marchandises sans autorisation pendant l'exposition". C'est en reprenant l'enquête en 2016 que, «naviguant» sur Internet, je tombe sur des clichés de la cour que je reconnais immédiatement. Certains renvoient au blog précédemment cité, d'autres à un site en lien avec la CNHI. Ces derniers sont présentés en plus grand nombre, seize au total, sans légende. Ils donnent un aperçu plus complet ${ }^{16}$ des graffiti et permettent de mieux apprécier leur valeur historique, qui ne repose plus à présent que 


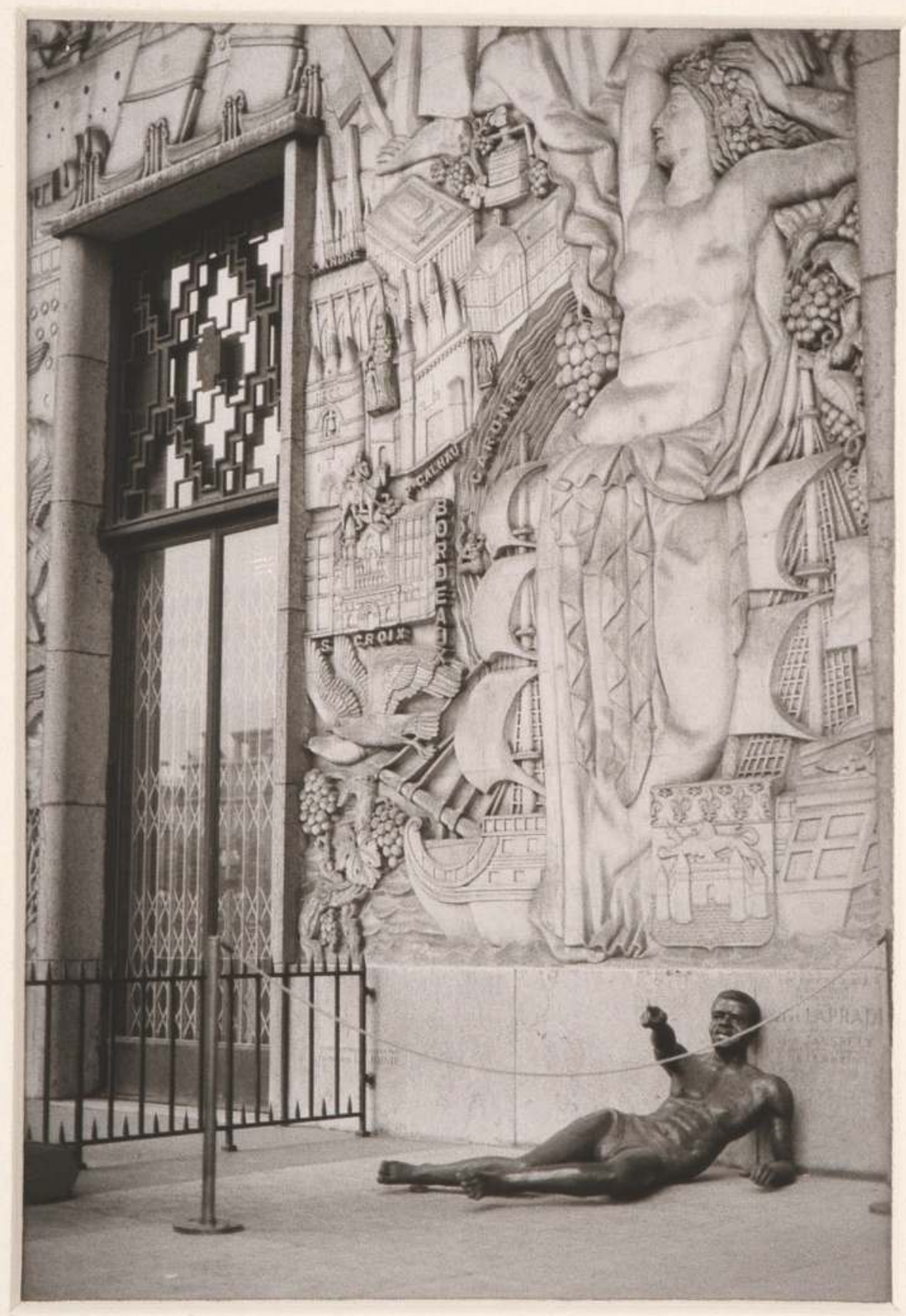




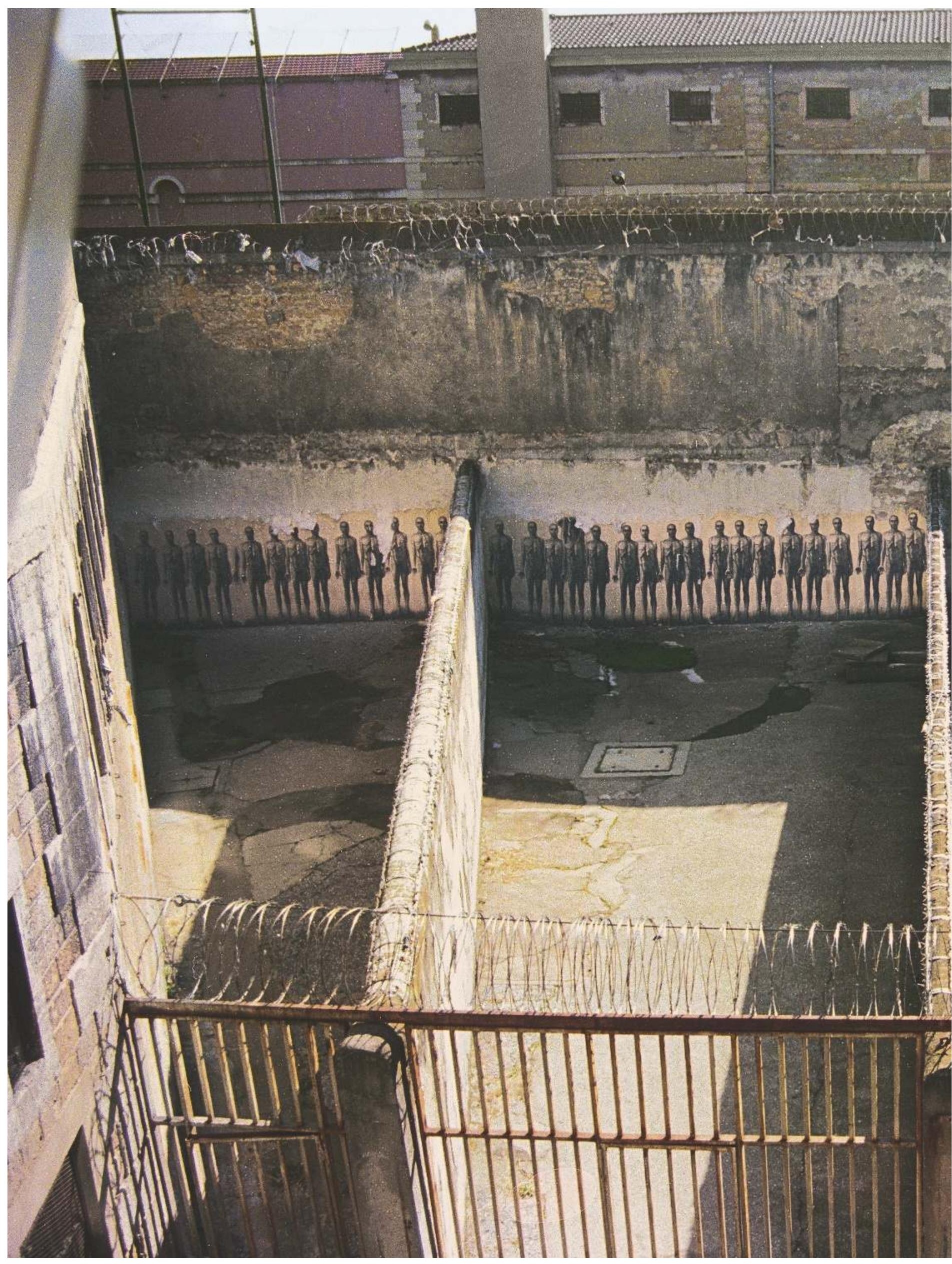




\section{double page \\ précédente}

fig. 7

Ernest Pignon Ernest,

Cours de la prison Saint

Paul à Lyon, 2012 @ Ernest

Pignon Ernest, ADAGP,

Paris 2016, avec l'aimable

autorisation de la galerie

Anne-Marie et Rolland

Pallade, Lyon.
17. Une exposition conçue par Laure Bulmé, France Poulain, Sylvie

Zaidman, Jean-Louis Breton, Dominique Pitte,

Benoît Pouvreau, Jean-

Claude Farcy, Jean-

Lucien Sanchez, Marc

Renneville, Jacky Tronel

et Jean-Claude Vimont

(coordination). Publiée le

15 octobre 2014 [en ligne],

disponible sur: https://

criminocorpus.org/fr/

musee/la-memoire-

des-murs/ (consulté le

12 mai 2016).

18. https://criminocorpus. $\mathrm{org} / \mathrm{fr} / \mathrm{musee} / \mathrm{la}-\mathrm{memoire-}$ des-murs/ (consulté le 9 mai 2016) sur quelques supports visuels. Les ressources numériques apportent, dans une certaine mesure, une solution à la sauvegarde de ces expressions graphiques qui retrouvent une place, certes immatérielle, au sein du Palais. Elles permettent au musée de repenser à son passé carcéral. Ainsi, le travail de mémoire autour des graffiti se poursuit à travers leur mise en ligne, leur archivage sur des sites Internet et l'ouverture des fonds constitués à un large public, qui peut entamer des recherches au gré de sa volonté.

On peut toutefois se demander si la raréfaction des commentaires, voire leur disparition, ne risque pas d'aboutir à terme à une réduction de la signification de ces images et plus encore à l'effacement, dans nos mémoires, de l'histoire qui leur est attachée, en particulier concernant la «cour des prisonniers » et les conditions de la détention de vendeurs à la sauvette en 1931. En ne documentant pas ou pas suffisamment les images diffusées, le virtuel ne joue-t-il pas contre la réalité in situ, au lieu d'en être un allié? Le toutpatrimonial et les nouvelles modalités de diffusion pourraient conduire à la perte de cet héritage visuel échouant à effectuer un véritable travail de transmission. On assisterait alors à un retournement du sens de la patrimonialisation: un patrimoine sans mémoire, sans souvenirs des événements qui se sont déroulés sur les lieux, à moins que ce ne soit la valeur graphique de cet art populaire qui ne devienne prépondérante? Un art sans archives, un livre de pierre uniquement pour ses images.

La plateforme Criminocorpus se distingue de ces autres blogs par ses ambitions archivistiques et par là historiques et scientifiques. Les graffiti y trouvent véritablement leur place. Soutenue par le CNRS et le ministère de la Justice, la plateforme centre son offre sur l'histoire de la justice, proposant en ligne des archives, des références d'ouvrages, des photographies, des vidéos (témoignages, visite d'un site) sur le thème de la prison. L'une des rubriques, «Musée d'histoire de la justice», présente une série d'expositions. Parmi elles, «La mémoire des murs ${ }^{17}$ » qui consacre de nombreuses pages aux "graffiti de prisons, de prisons politiques et militaires, de colonies pénitentiaires et de camps d'internement ${ }^{\mathbf{1 8}}$ ». Cette exposition coordonnée par l'historien Jean-Claude Vimont est née d'une initiative pédagogique au sein du master professionnel «valorisation du patrimoine » à l'université de Rouen. La richesse du travail a conduit à déposer les résultats sur le site de Criminocorpus, leur donnant une plus grande visibilité, et a encouragé les initiatives de collecte, académiques ou amateurs, pour poursuivre l'inventaire. Chacun peut s'improviser à son heure historien, et collaborer à l'enrichissement des fonds du site. Cette sauvegarde pousse à la loi du nombre, à l'exhaustivité, à l'accumulation, nourrissant les séries documentaires, les préservant tout en les donnant à voir. Le musée de l'Histoire de l'immigration pourrait ainsi publier sur criminocorpus.org les documents se rapportant à la «cour des prisonniers». Ce sont aujourd'hui plus de trente sites carcéraux qui sont répertoriés et documentés. Une cartographie à l'échelle nationale est en train de se dessiner progressivement, avec une mise à jour du corpus qui enrichit la base de données existante. On y retrouve la prison de Brignoles, celle de Sainte-Anne à Avignon et d'autres encore. Pour sept prisons, les photographies de graffiti ont la part belle: elles défilent en ruban avec leurs légendes et offrent la possibilité de s'arrêter sur le détail de ces réalisations graphiques, de les étudier précisément 
comme des archives ou simplement de les regarder comme des œuvres photographiques.

Paradoxalement, ces ressources numériques nous renvoient à la fragilité des sources matérielles. Elles n'ont cependant pas uniquement pour fonction d'éviter leur perte et de lutter contre leur oubli. Les rassembler en un même espace fonde en soi un nouveau «lieu de mémoire». Là, s'élabore la valeur des graffiti et se fabrique aussi, grâce aux outils informatiques, leur rôle mémoriel et plus encore mémorial. La dimension patrimoniale et artistique de ces écritures s'y trouve validée et ainsi renforcée. Le dispositif technique couplé à un environnement scientifique contribue à instaurer une distance critique avec l'objet-graffiti, le transformant en objet de curiosité et/ou objet de recherche qui permet la transmission de son histoire et aide à composer avec ces «héritages encombrants " (Djament-Tran 2013).

\section{Les graffiti aux prises avec l'histoire}

La valorisation des productions murales du passé ne se situe pas toujours là où l'on s'y attendrait et reflète surtout la relation que les institutions (prisons, collectivités locales, musées, etc.) entretiennent avec les traces de notre histoire, et surtout de la leur. Jean-Yves Boursier explique que les «traces ont toujours intéressé les hommes dans la mesure où elles matérialisent ce qui a disparu, lui donnent une image, permettent de se le représenter, de l'étudier, de se souvenir, de commémorer, de montrer une évolution en remontant le temps» (Boursier 2002). Elles sont «ce qui nous reste». Quand le temps n'a pas effacé les graffiti sur les murs des lieux de détention, ces actes graphiques - «cicatrices murales - conservent un rôle mnémonique. Ils sont des repères matériels et visuels aidant à l'ancrage physique qui participe à la construction d'une mémoire collective (Halbwachs 1968 [1949]). Ils sont une trace tangible d'une pensée et d'une action, qui ont valeur de témoignage (Hameau 2013a) et, à ce titre, se placent du côté de l'archive. Ils renvoient à des strates d'histoires, parfois à leur toute dernière couche, ce qui transforme les murs en palimpseste, conservant uniquement la mémoire des dernières écritures. Dans tous les cas, les supports muraux ainsi marqués sont et restent les témoins et la preuve vivante de cette occupation humaine liée à des circonstances politiques, religieuses ou sociales.

Ces «trésors" archivistiques se présentent comme des aveux de I'histoire qui en disent trop, voire beaucoup trop sur certains agissements passés. Le déni est l'une des réactions possibles face au poids de ces aveux. Que faire alors du devoir de mémoire? Comment, dans ce contexte précis, la patrimonialisation de ces graffiti est-elle possible et quel sens donner à ce processus?

Selon Daniel Fabre, «[...] la condition carcérale ne suffit pas à qualifier de façon stable un "art", en revanche, comme elle aboutit à un marquage dense des lieux par les prisonniers eux-mêmes, elle produit un monde de signes forcément obscurs, plus ou moins codés et toujours liés à une situation pathétique que l'histoire magnifie. Cette triple alliance de l'énigme, de la souffrance et du passé contribuera fortement à la pleine conversion du palimpseste des prisons en bien commun, en patrimoine, source d'émotion, 


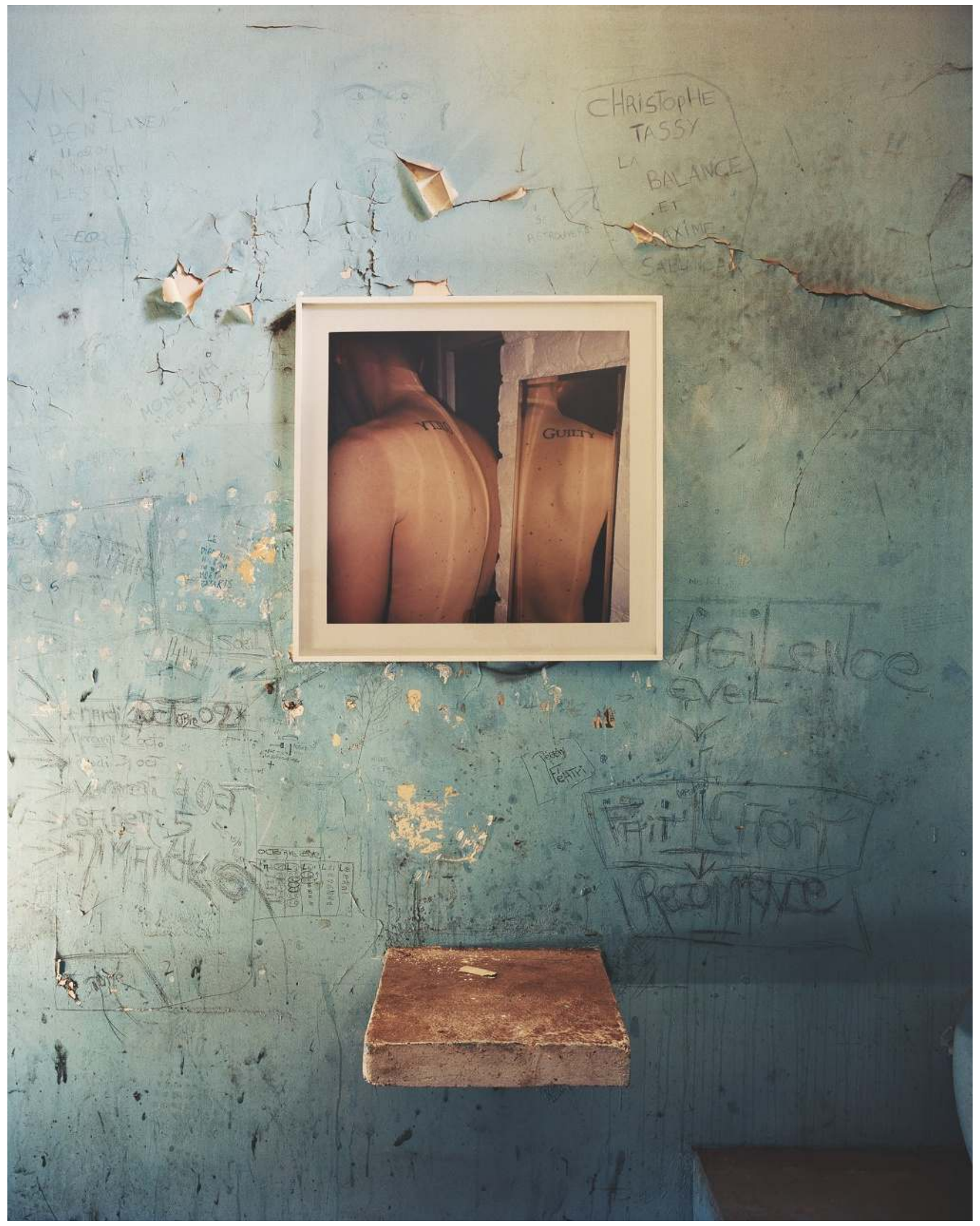


d'émerveillement, de dépaysement et d'identification » (Fabre 2007 : 54, traduction de Daniel Fabre). Pour que le processus de reconnaissance puisse être engagé, il faut - nous explique Sophie Wahnich - une prise en charge institutionnelle: «Cette soustraction au temps engage une politique qu'on pourrait appeler une politique de l'archive, au sens non pas seulement des documents écrits, conservés dans des fonds d'archives, mais au sens où il y a là une institution qui déclare que sa fonction est d'archiver un événement qui doit faire loi pour l'humanité. » (Wahnich 2011 : 51)

Avec ou sans les murs in situ, la constitution d'un «patrimoine négatif » (Wahnich 2011), à la fois archive et art à protéger, fait sortir de l'ombre les prisonniers. La patrimonialisation des gratiffi carcéraux n'a pas pour objectif de réenchanter le monde carcéral ou de magnifier la «beauté du mort», mais bien de faire prendre conscience des faits de l'histoire, d'en mesurer toute la portée politique et sociale et de transmettre ce message aux générations futures. En cela je rejoins les propos de Sophie Wahnich quand elle avance: «[...] plus que les ruines et les documents, la conservation des traces dites authentiques permettrait de produire une transmission de ce qui est difficilement imaginable ou douloureusement imaginable. » (Wahnich 2011: 51)

II reste que le contexte contemporain de cette transmission n'est pas sans poser quelques questions. Comme le souligne Henri-Pierre Jeudy, aujourd'hui l'injonction à ne pas oublier est si forte qu'elle engendre parfois un sentiment de culpabilité dès lors que le devoir n'est pas accompli (Jeudy 2008 [2001]). Le devoir de mémoire se transforme en une obligation de mémoire et par conséquent invite à tout garder, jusqu'à l'inflation et le brouillage des signes. De même, «la conservation patrimoniale se charge du dépôt des souvenirs et nous délivre du poids des responsabilités infligées à la mémoire. [...] Seulement ce travail de deuil, s'il est trop bien accompli, ne risque-t-il pas à son tour de provoquer un excès de tranquillité des mémoires collectives? » (ibid. : 7).

À travers les arbitrages - patrimonialiser in situ ou virtuellement, définir comme un art ou abandonner, témoigner ou passer sous silence -, nous saisissons les difficultés souvent rencontrées pour «faire avec» l'histoire des lieux. Si ces graffiti carcéraux demeurent encore «en péril» (Vimont 2008), c'est sans doute parce que, associés à un lourd passé, ils inspirent de la répulsion, provoquée par la «morbidité » qu'ils convoquent (Jeudy 2008 [2001]). En cela, les graffiti, œuvres ordinaires, sont et restent des «archives sensibles», et ce d'autant plus qu'elles sont marquées par une dimension politique.

\section{ci-contre}

fig. 8

Douglas Gordon, Guilty (Tatoo for Reflexion), 1997 photographie couleur. Donation Yvon Lambert à l'État français / Centre national des arts plastiques / Dépôt à la Collection Lambert. Installation dans une cellule de la prison Sainte-Anne à l'occasion de l'exposition La disparition des lucioles organisée en 2014 par la Collection Lambert, Musée d'art contemporain, Avignon @ ADAGP, Paris,

2016 (C) Photographie

François Halard. 
Bibliographie

Benavente, José

Antoniao et al.,

2004 " Les graffiti des prisons du Bas-Aragon (Espagne): un cas exemplaire de patrimonialisation ", Le Monde alpin et rhodanien 1-2: 131-144.

\section{Berthet, Frédérique,} photographies Dal Molin, Rémy

2015 "La prison Sainte-Anne d'Avignon. D'ouvertures en fermetures", Vacarme 72: 112-129.

\section{Boursier, Jean-Yves}

2002 «La mémoire comme trace des possibles", Socioanthropologie 12 [en ligne], disponible sur: http://socioanthropologie.revues.org/145 (consulté le 12 avril 2016).

\section{Calet, Henri}

1993 [1945] Les Murs de Fresnes. Paris, Viviane Hamy.

\section{Candau, Joël et Hameau,} Philippe (dir.)

2004 Cicatrices murales. Les graffiti de prison (Le Monde alpin et rhodanien 1-2).

Certeau (de), Michel, en collaboration avec Julia, Dominique et Revel, Jacques

1993 [1974] «La beauté du mort », in Michel de Certeau, La Culture au pluriel. Paris, Seuil.

\section{Charuty, Giordana}

2008 "Les scènes du texte", in Brigitte Baptandier et Giordana Charuty, Du corps au texte. Approches comparatistes. Nanterre, Société d'ethnologie: 25-41.

\section{Clemente, Pietro (dir.)}

2007 Prima persona. Percorsi autobiografici 17.

À l'ombre des murs palimpsestes. Par Anne Monjaret

\section{Debary, Octave}

$\mathbf{2 0 0 0}$ "L'écomusée est mort, vive le musée ", Publics et Musées 17-18: $71-82$.

\section{Debray, Régis}

1999 "Trace, forme ou message? ", Les Cahiers de Médiologie 7 : 27-44.

\section{Demetrio, Duccio}

2007 "Crepe nel muro", Prima persona. Percorsi autobiografici $17: 71-76$.

\section{Djament-Tran, Géraldine}

2013 «Patrimoine urbain, lieux de mémoire et fonctions de capitale à Berlin, de l'unité allemande à la réunification ", Revue géographique de l'Est 53 (3-4) [en ligne], disponible sur: http://rge.revues. org/5034 (consulté le 25 avril 2016).

Eidelman, Jacqueline, Monjaret, Anne, Roustan, Mélanie, photographies de

Bernard Plossu

2002 MAAO. Mémoires. Paris, Marval.

2003 «MAAO, mémoire d'une organisation ", Culture \& Musées 2: 101-127.

\section{Fabre, Daniel}

2007 "l libri di pietra», Prima persona. Percorsi autobiografici 17 : 49-55.

\section{Fabre, Daniel et Iuso,} Anna (dir.)

2010 Les monuments sont habités. Paris, Éditions de la Maison des sciences de l'homme.

\section{Foucault, Michel}

1975 Surveiller et punir: naissance de la prison. Paris, Gallimard.

\author{
Halbwachs, Maurice \\ 1968 [1949] La Mémoire collective. \\ Paris, PUF.
}

\section{Hameau, Philippe}

2004 "Traces et espaces à la prison de Brignoles ", Le Monde alpin et rhodanien 1-2: 13-28.

2008 "Une œuvre autobiographique en milieu carcéral ", Ethnologie française $1:$ 151-162.

2009a "Des mots qui ne peuvent scier des barreaux", Cultures \& Sociétés. Sciences de l'homme 10: 64-69.

2009b «Le temps de la réclusion », in Joël Candau (dir.), Temps en partage: ressources, représentations, processus, actes du $129^{\circ}$ Congrès national des sociétés historiques et scientifiques (Besançon, 2004). Paris, Éditions du CTHS : 57-68.

2013a "Graffiti de prison et patrimoine", Vestighe 2 : 38-47.

2013b «L'idéal de la paix et les réalités de la guerre à travers les graffiti de la prison de Brignoles ", in Simone Mazauric (dir.), Écrire la guerre, écrire la paix, actes du $136^{\circ}$ Congrès national des sociétés historiques et scientifiques (Perpignan, 2011). Paris, Éditions du CTHS : 85-94.

\section{Jeudy, Henri-Pierre \\ 2008 [2001] La Machinerie patrimoniale. Paris, Circé.}

\section{Leschiutta, Pierpaolo,}

1996 "Palimsesti del carcere»: Cesare Lombroso e le scritture proibite. Naples, Liguori Editore.

\section{Levi, Primo}

1987 [1958] Si c'est un homme, trad. de l'italien par Martine Schruoffeneger. Paris, Julliard.

\section{Lombroso, Cesare}

1888 Palimsesti del carcere. Raccolta unicamente destinata agli uomini di scienza. Turin, Fratelli Bocca.

\section{Mézil, Éric (dir.)}

2014 La Disparition des lucioles, catalogue d'exposition (Avignon, prison Saint-Anne). Arles, Collection Lambert/Actes Sud.

\section{Monjaret, Anne (dir.)}

2005 Fermetures: crises et reprises (Ethnologie française 4).

\section{Monjaret, Anne}

2004 "La cour des "prisonniers" graffiti et métaphore carcérale dans un musée parisien ", Le Monde alpin et rhodanien 1-2: 77-88.

\section{Murphy, Maureen}

2007a Un palais pour une cité: du musée des Colonies à la Cité nationale de l'histoire de l'immigration. Paris, RMN/CNHI.

2007b "La CNHI au palais de la Porte Dorée ", Hommes \& Migrations 1267: 44-55.

\section{Pitrè, Giuseppe et} Sciascia, Leonardo

1999 [1977] Urli senza suono. Graffiti e disegni dei prigionieri dell'Inquisizione. Palerme, Sellerio.

\section{Portigliatti Barbos, Mario}

2004 "Les "palimpsestes" lombrosiens ", Le Monde alpin et rhodanien 1-2: 125-130.

\section{Pignon-Ernest, Ernest}

2014 Pignon-Ernest. Prisons, catalogue d'exposition, préface Gérard Mordillat. Paris, galerie Lelong.

\section{Saurier, Delphine (dir.)}

2016 Entre les murs / Hors les murs. Culture et publics empêchés (Culture \& Musées 26).

\section{Stewart-Steinberg, Suzanne}

2007 The Pinocchio Effect: on Making Italians (1860-1920). Chicago, Universty of Chicago Press. 
Truchi, Sandrine

2004 "Temps passé et temps ressenti: les graffiti de l'ancienne prison de Brignoles ", Le Monde alpin et rhodanien 1-2: 29-46.

\section{Vimont, Jean-Claude}

2008 " Graffiti en péril ? ", Sociétés \& Représentations 25: 193-202.

\section{Wahnich, Sophie}

2011 «L'impossible patrimoine négatif », Les Cahiers Irice 7: 47-62. page 164 et ci-contre Ernest Pignon Ernest, Cours de la prison Saint-Paul à Lyon, 2012 (c) Ernest Pignon Ernest, ADAGP Paris 2016, avec l'aimable autorisation de la galerie Anne-Marie et Rolland Pallade, Lyon.
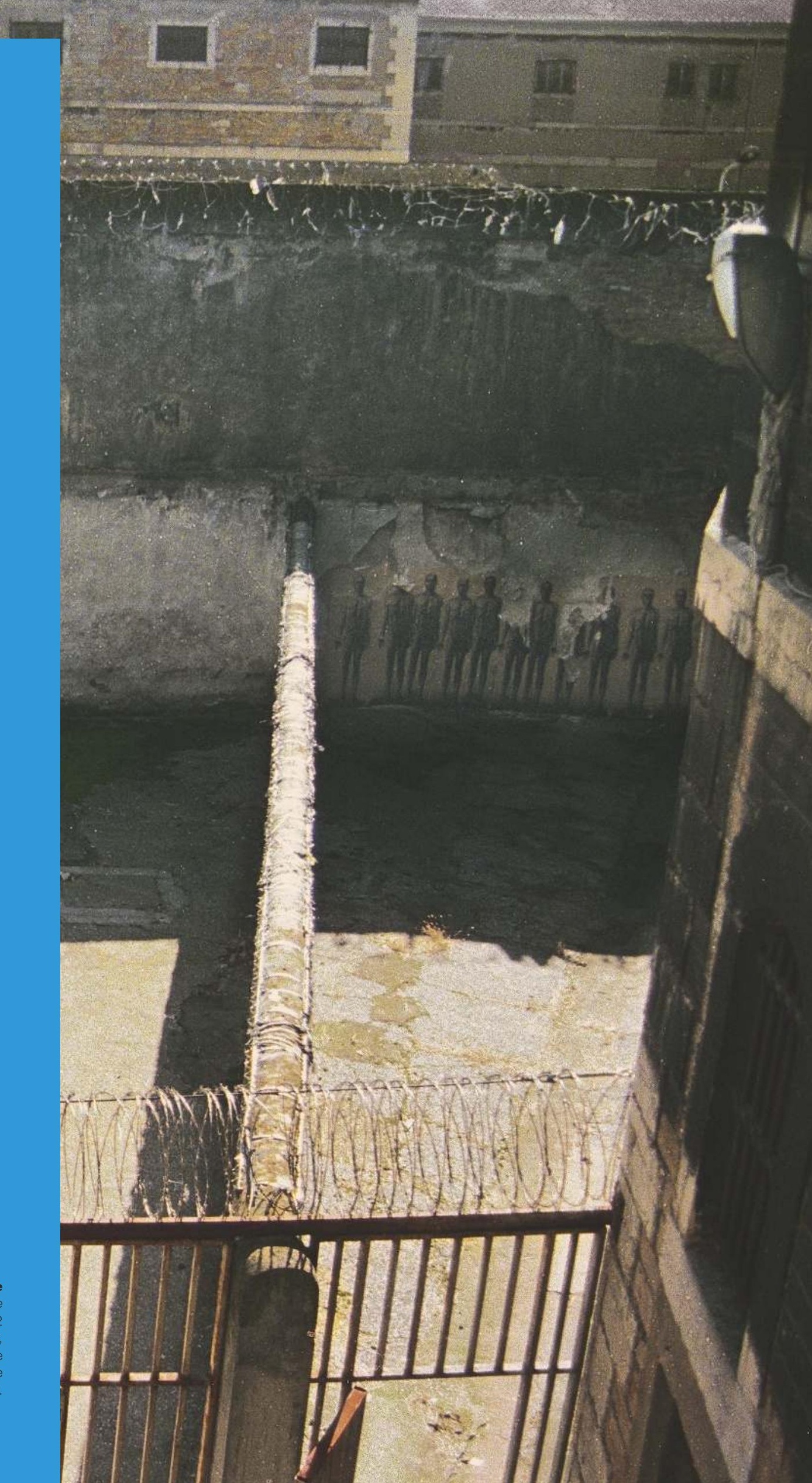